\title{
Research Paper \\ Factors Related to Frailty Among Older Adults in Khuzestan, Iran
}

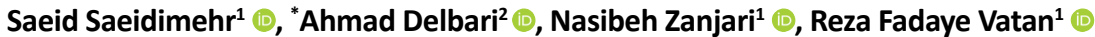

1. Department of Aging, University of Social Welfare and Rehabilitation Sciences, Tehran, Iran

2. Aging Research Center, University of Social Welfare and Rehabilitation Sciences, Iran.

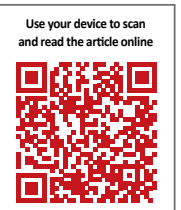

Citation: Saeidimehr S, Delbari A, Zanjari N, Fadaye Vatan R. [Factors Related to Frailty Among Older Adults in Khuzestan, Iran (Persian)]. Iranian Journal of Ageing. 2021; 16(2):202-217. https://doi.org/10.32598/sija.16.2.1600.1

doi https://doi.org/10.32598/sija.16.2.1600.1

Key words:

Older adult, Frailty,

Related factors,

Khuzestan

\begin{abstract}
A B STRACT
Objectives Frailty syndrome is one of the most important geriatric syndromes that imposes high psychological and financial costs on the elderly, their families and health system. This study aims to determine the factors associated with frailty in older adults living in Khuzestan province of Iran

Methods \& Materials This is a cross-sectional study conducted in 2019 on 540 older adults in Khuzestan province who were selected by a multistage random sampling method. Data collection tools include a demographic form, Deficit accumulation Frailty Index, Wax's Social Support Scale and the Geriatric Depression Scale. Collected data were analyzed using Spearman correlation test, chisquare test and sequential logistic regression analysis.

Results The Mean \pm SD age of participants was $72.61 \pm 8.72$ years and $65.6 \%$ were women. Around $10.4 \%$ were frail and $25.6 \%$ were pre-frail. Frailty was higher among older women with higher age, lower education, without social support, with depression, living alone, single, with a history of taking high number of medications, and with a history of hospitalization $(\mathrm{P}<0.001)$. Frailty was lower in older men with lower age, a history of taking less than 5 medications, no depression, and high social support $(P<0.005)$.

Conclusion The consequences of frailty syndrome can be reduced by early identification of frail older adults and using appropriate interventions based on risk factors such as polypharmacy and low social support.
\end{abstract}

\section{Extended Abstract}

\section{Introduction}

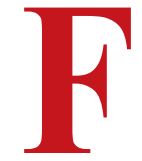

railty is a clinical syndrome defined by impaired physiological systems, reduced reserve capacity, and decreased ability to cope with stress. It is associated with complications such as falls, delirium, and death. It is influenced by several factors including demographic factors (age, gender, education, income, marital status), medical history (presence of diseases such as heart failure, heart attack, cancer, diabetes), social status (dissatisfaction with life, poor quality of life, accidents in life, low income) and psychological status. In Iran, few studies have been conducted to investigate the factors associated with frailty syndrome. The aim of this study was to investigate the factors related to frailty syndrome in Khuzestan province of Iran.

\section{Methods}

The cross-sectional study was conducted on 540 older adults aged $\geq 60$ years in Khuzestan province who were selected by multi-stage random sampling method. Data

\section{* Corresponding Author:}

Ahmad Delbari, PhD.

Address: Aging Research Center, University of Social Welfare and Rehabilitation Sciences, Iran.

Tel: +98 (61) 34457292

E-mail: ahmad_1128@yahoo.com 
collection tools were (a) a demographic form surveying age, gender, education, marital status, living arrangements, income, number of used medications, history of hospitalization, and smoking, (b) Deficit accumulation frailty index introduced by Searle et al. in 2008 and expanded by Rockwood et al. in 2011. It has 40 items rated as 0 or 1 . The index value is calculated by dividing the sum of deficit scores by the number of deficits. A person with zero score is considered as a healthy older person, and while the score $\geq 0.75$ indicates frailty; (c) Geriatric depression scale developed by Yesavage [21] and has 30 items. The psychometric properties of its Persian version have been evaluated by Rockwood et al. [22]. Based on the cut-off point 8, a score of 1-5 indicates mild depression, 9-11 indicates moderate depression, and 12 or higher indicates severe depression; (d) Social Support scale developed by Wax et al. in 1986. Its Persian version was prepared and localized by Ebrahimi et al. [27]. Data analysis was performed in SPSS v. 22 software. Statistics such as frequency, percentage, mean and standard deviation were used to describe the obtained data. Nonparametric tests such as Chi-square, Spearman correlation and sequential logistic regression were used to examine the relationship between the main variables of the study.

Significance level for all tests was set at 0.05 .

\section{Results}

The Mean \pm SD age of participants was $72.61 \pm 8.72$ years. Of 540 participants, 65.6 were women; $64 \%$ were married;
$79 \%$ had a diploma or lower degree, $57 \%$ had moderate income level; 57\% took less than 5 medications per day; 30\% had a history of hospitalization in the past year, and $6.7 \%$ were living alone; and $46.7 \%$ had no history of smoking. Moreover, 10.6 were frail, 25.6 were pre-frail and 63.1were not frail. Also, the percentage of older women with frailty was higher than that of frail older men. In terms of depression, $59 \%$ were in the normal group and $4.8 \%$ were in the severe depression group. In terms of social support, 37.4\% had poor social support and only $19.4 \%$ had high social support. Frailty had a statistically significant relationship with age, gender, history of hospitalization, living arrangements, social support and depression (Table 1). The prevalence of frailty increases with aging, in females, having lower education, increased hospitalization, living alone, decreased social support, and depression. According to regression analysis results, the level of frailty was lower in older men, younger older adults, the elderly taking less than 5 medications per day, those without depression and with high social support.

\section{Discussion and Conclusion}

In this study, the prevalence of frailty syndrome was higher among older women, those with lower education, without social support, depression, living alone, with a history of taking more medications, and with a history of hospitalization. This group of older people need more and timely care to reduce the social, medical and psychological conse-

Table 1. Frailty prevalence based on sociodemographic characteristics, social support and depression among the elderly

\begin{tabular}{|c|c|c|c|c|c|c|}
\hline \multirow{2}{*}{\multicolumn{2}{|c|}{ Variables }} & \multicolumn{3}{|c|}{ No. (\%) } & \multirow{2}{*}{$\chi^{2}$} & \multirow{2}{*}{$\mathbf{P}$} \\
\hline & & Frail & Pre-frail & Not frail & & \\
\hline \multirow{2}{*}{ Gender } & Female & $42(7.5)$ & $88(16.3)$ & $224(41.5)$ & \multirow{2}{*}{13.73} & \multirow{2}{*}{0.001} \\
\hline & Male & $14(2.9)$ & $55(10.2)$ & $117(21.5)$ & & \\
\hline \multirow{3}{*}{ Age } & $60-74$ & $12(3.9)$ & $64(20.8)$ & $232(75.3)$ & \multirow{3}{*}{130.36} & \multirow{3}{*}{$<0.001$} \\
\hline & $78-84$ & $28(17.8)$ & $54(34.4)$ & $75(47.8)$ & & \\
\hline & $>85$ & $37(49.3)$ & $21(28.0)$ & $17(22.7)$ & & \\
\hline \multirow{6}{*}{ Educational level } & Illiterate & $10(22.2)$ & $12(26.7)$ & 23(51.1) & \multirow{6}{*}{17.214} & \multirow{6}{*}{0.07} \\
\hline & $\begin{array}{l}\text { Primary school } \\
\text { education }\end{array}$ & 10(14.9) & $17(25.4)$ & 40(59.7) & & \\
\hline & $\begin{array}{l}\text { Middle school } \\
\text { education }\end{array}$ & $20(20.6)$ & $25(25.8)$ & $52(53.6)$ & & \\
\hline & $\begin{array}{c}\text { High school educa- } \\
\text { tion }\end{array}$ & 17(16.0) & $33(31.1)$ & $56(52.8)$ & & \\
\hline & Diploma & $12(10.5)$ & $23(20.2)$ & 79(69.3) & & \\
\hline & Academic degree & $8(7.2)$ & $29(26.1)$ & $74(66.7)$ & & \\
\hline
\end{tabular}




\begin{tabular}{|c|c|c|c|c|c|c|}
\hline \multirow{2}{*}{\multicolumn{2}{|c|}{ Variables }} & \multicolumn{3}{|c|}{ No. (\%) } & \multirow{2}{*}{$\chi^{2}$} & \multirow{2}{*}{$\mathbf{P}$} \\
\hline & & Frail & Pre-frail & Not frail & & \\
\hline \multirow{3}{*}{ Social support } & Poor & $45(22.3)$ & $52(25.7)$ & $105(52.0)$ & \multirow{3}{*}{18.447} & \multirow{3}{*}{0.001} \\
\hline & Moderate & $24(10.3)$ & $59(25.3)$ & $150(64.4)$ & & \\
\hline & High & $8(7.6)$ & $28(26.7)$ & $69(65.7)$ & & \\
\hline \multirow{2}{*}{ Depression } & No & $33(10.4)$ & 59(18.6) & $226(71.1)$ & \multirow{2}{*}{39.494} & \multirow{2}{*}{$<0.001$} \\
\hline & Yes & $44(19.8)$ & $80(36.0)$ & $98(44.1)$ & & \\
\hline \multirow{4}{*}{ living arrangement } & Alone & $11(30)$ & $9(25)$ & $16(44.4)$ & \multirow{4}{*}{-0.215} & \multirow{4}{*}{$<0.001$} \\
\hline & With spouse & $31(25.4)$ & $35(28.7)$ & $56(45.9)$ & & \\
\hline & With family & $17(11.1)$ & $34(22.2)$ & $102(66.7)$ & & \\
\hline & With children & $18(7.9)$ & $61(26.6)$ & $150(65.5)$ & & \\
\hline \multirow[b]{2}{*}{ Marital status } & Single & $45(22.3)$ & $49(24.3)$ & $108(53.5)$ & \multirow[b]{2}{*}{33.99} & \multirow[b]{2}{*}{$<0.001$} \\
\hline & Married & $32(9.5)$ & $90(26.6)$ & $116(63.9)$ & & \\
\hline \multirow{6}{*}{$\begin{array}{l}\text { Head of household } \\
\text { income (Tomans) }\end{array}$} & $<1$ million & 13(18.3) & $20(28.2)$ & $38(53.5)$ & \multirow{6}{*}{11.27} & \multirow{6}{*}{0.336} \\
\hline & 1-1.5 million & $15(17)$ & $27(30.7)$ & $46(52.3)$ & & \\
\hline & 10.5-2 million & $25(18.1)$ & $30(21.7)$ & $83(60.1)$ & & \\
\hline & 2-2.5 million & 11(11.6) & $21(22.1)$ & $63(66.3)$ & & \\
\hline & 2.5-3 million & $6(8)$ & $21(28)$ & $48(64)$ & & \\
\hline & $>3$ million & $7(9.6)$ & $20(27.4)$ & $46(63)$ & & \\
\hline \multirow{3}{*}{$\begin{array}{l}\text { Number of taken } \\
\text { medications }\end{array}$} & No income & $2(3.7)$ & 19(35.2) & $33(61.1)$ & \multirow{3}{*}{0.158} & \multirow{3}{*}{$<0.001$} \\
\hline & $1-5$ & $22(8.6)$ & $67(26.2)$ & $168(65.2)$ & & \\
\hline & $>5$ & $53(23)$ & $53(23)$ & 124(53.9) & & \\
\hline \multirow{2}{*}{$\begin{array}{l}\text { History of hospital- } \\
\text { ization }\end{array}$} & Yes & $55(33.3)$ & $30(18.2)$ & $80(48.5)$ & \multirow{2}{*}{71.14} & \multirow{2}{*}{$<0.001$} \\
\hline & No & $22(5.9)$ & $109(29.1)$ & $244(65.1)$ & & \\
\hline \multirow{2}{*}{ Smoking } & Yes & 15(9.7) & $47(30)$ & $93(60)$ & \multirow{2}{*}{0.04} & \multirow{2}{*}{0.35} \\
\hline & No & $62(16.1)$ & $92(23.9)$ & $231(60)$ & & \\
\hline
\end{tabular}

SALMAND

quences of frailty syndrome. Screening and early detection of this syndrome among the elderly for early interventions prevent them from entering the full level of frailty. The present study is the first study in Iran that investigates the factors related to frailty in the elderly. It is recommended that national plans for elderly health assessment be performed to identify and screen, treat and care for frail older adults in other provinces of Iran. Moreover, support facilities for frail older adults, especially older women, should be provided by increasing services and access to health facilities for the treatment of their diseases.

\section{Ethical Considerations}

\section{Compliance with ethical guidelines}

This study has an ethical approval obtained from the Ethics Committee of Tehran University of Social Welfare and Rehabilitation Sciences (Code: IR.USWR.REC.1398.365). All ethical principles are considered in this article. The participants were informed about the purpose of the research and its implementation stages. They were also assured about the confidentiality of their information and were free to leave the study whenever they wished, and if desired, the research results would be available to them. 


\section{Funding}

This research did not receive any specific grant from funding agencies in the public, commercial, or non-profit sectors.

Authors' contributions

All authors contributed equally in preparing this article.

\section{Conflicts of interest}

The authors declare no conflict of interest

\section{Acknowledgements}

The authors would like to thank the Vice-Chancellor for Health of Ahvaz Jundishapur University of Medical Sciences, the managers of Health Centers in Ahvaz, Mahshahr Port, Masjed Soleiman, Susangard and Dezful cities, and the participants for their support and cooperation. 
This Page Intentionally Left Blank 


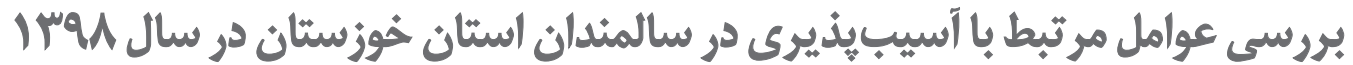

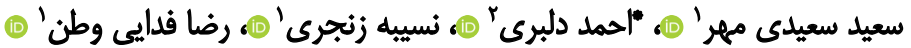

ا. بروه آموزشى سالمئدى، دانشكاه علوم بهزيستى و توانبخشى، تهران، ايران.

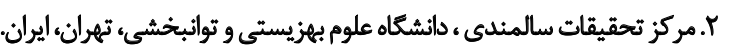

\begin{abstract}
حكSد

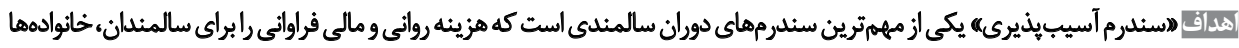

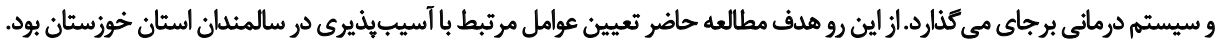

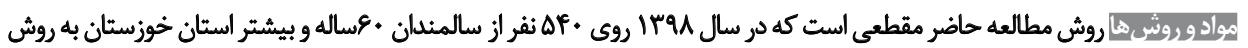

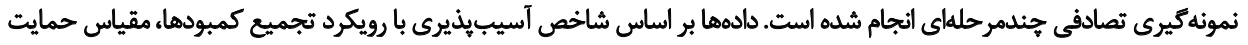

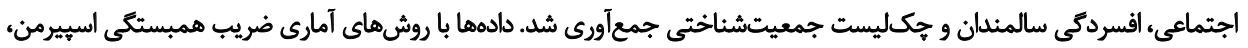

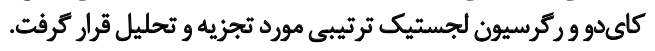

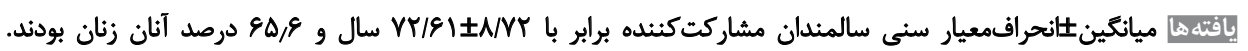

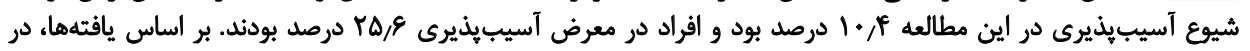

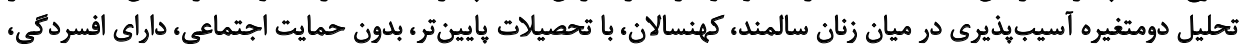

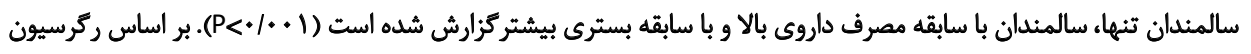

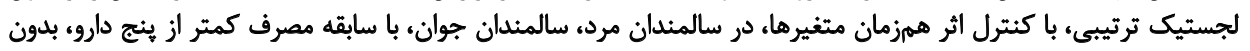

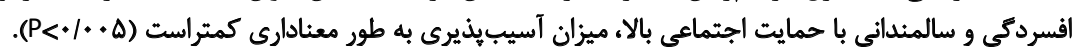

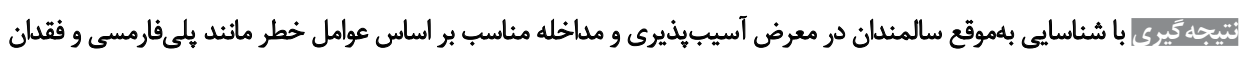

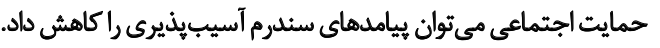

تاريخ دريافت: ال مرداد

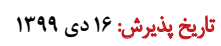

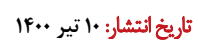

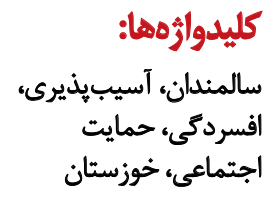

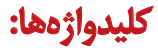

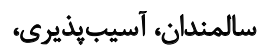

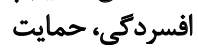

اجتماعى، خوزستان حمان

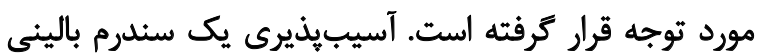

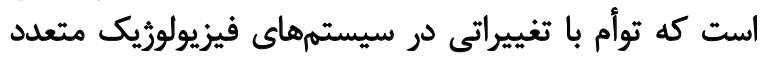

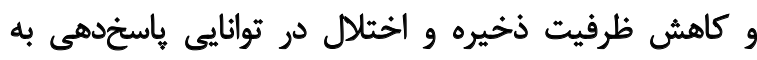

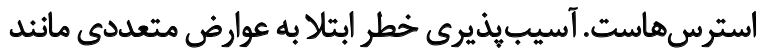

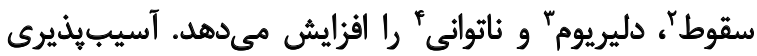

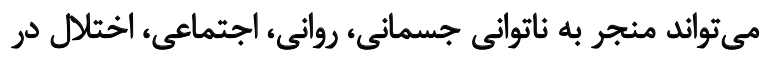

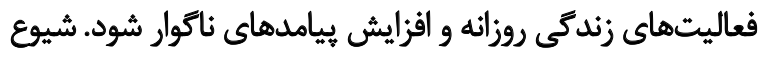

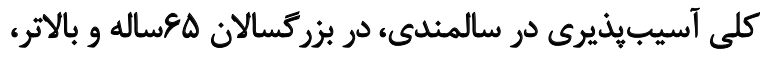

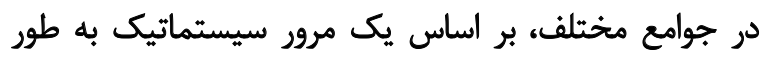

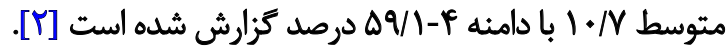
2. Falls
3. Delirium
4. Disability

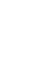

$$
\text { ㄷ. }
$$

: نويسئدة مسينول:

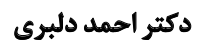

نشاني: تهران، دانشكاه علوم توانبخشى و سلامث اجتماعي، مركز تحقيقات سالمندى.

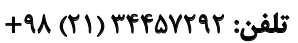
يست الكترونيكي: ahmad_1128@yahoo.com 


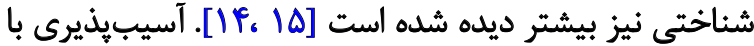

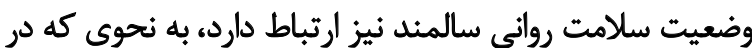

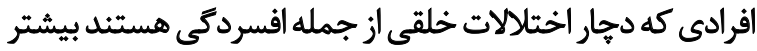

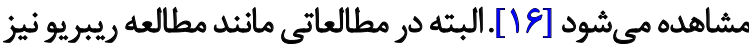

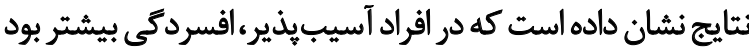

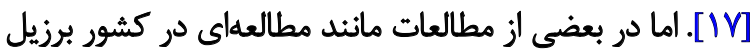

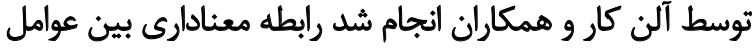

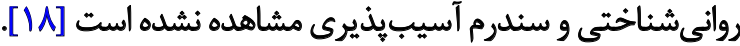

تحقيقات متعددى در حيطه عوامل مرتبط با آسيبذيذيرى

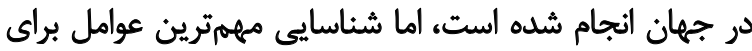

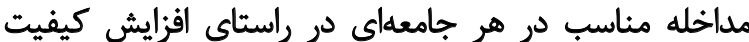

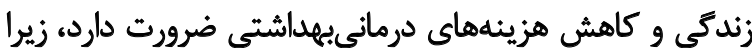

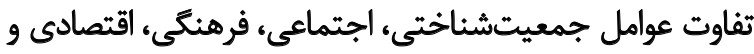

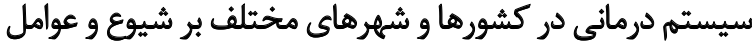

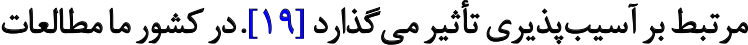

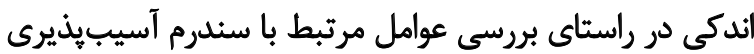

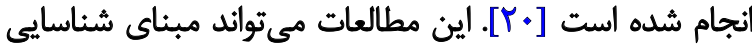

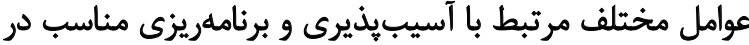

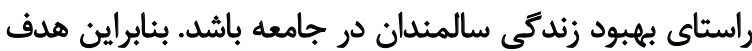

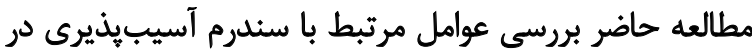
استان خوزستان است. روش مطالعه

يروهش حاضر به روش مقطعى انجام شده است. جامعه آمارى

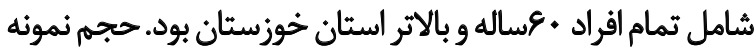

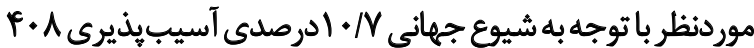

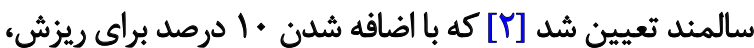

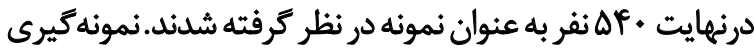

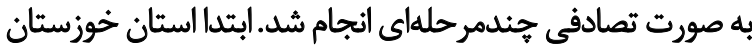

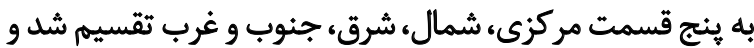

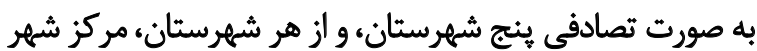

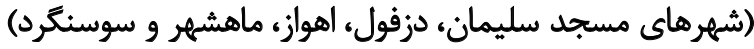

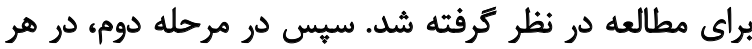

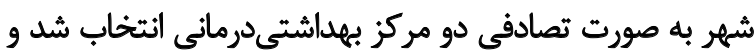

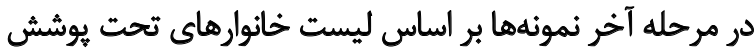

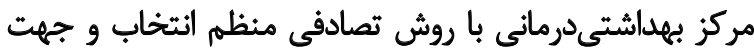

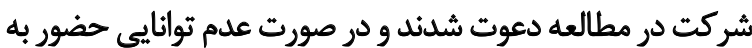

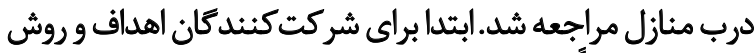

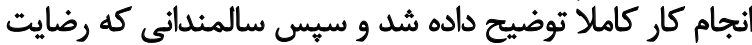
براي شركت در طرح را داشتند، وارد مطالعه شدند.

ملاكهاى ورود به مطالعه شامل سن بالاى •ع سال، رضايت

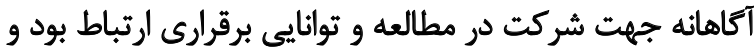

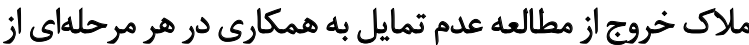

سندرم آسيبذيذيرى در دوران سالمندى تحت تأثير عوامل

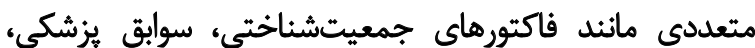

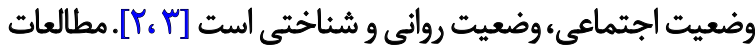

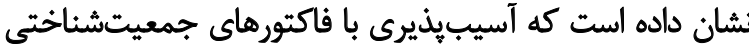

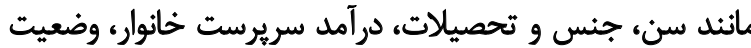

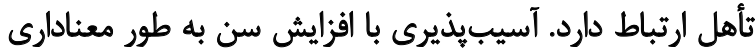

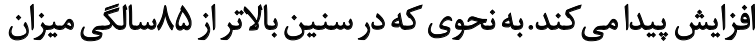

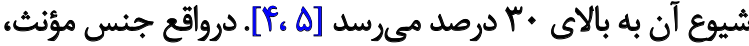

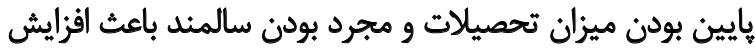

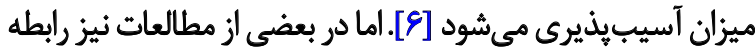

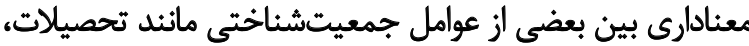

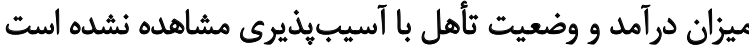

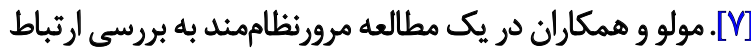

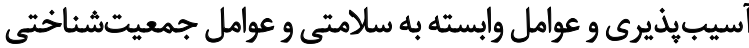

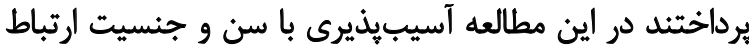

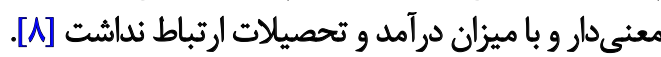
در مورد سوابق يزشكى سالمند نيز بعضى از تحقيقات نشان

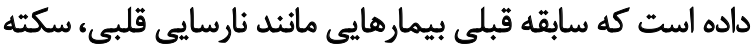

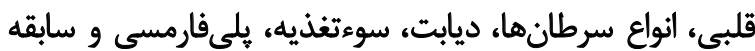

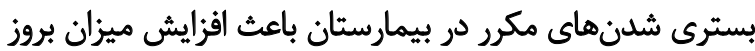

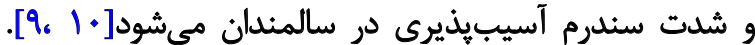

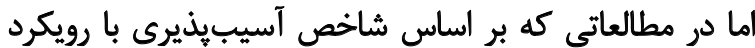

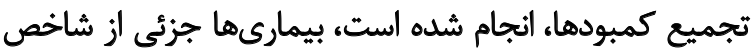

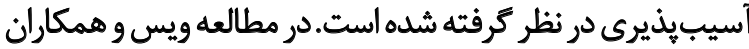

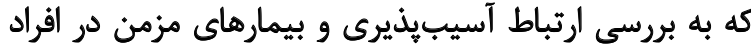

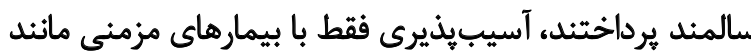

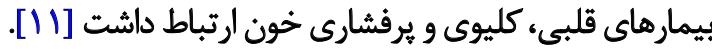
وضعيت اجتماعى سالمندان نيز يكى از مهمثرين عواملى است

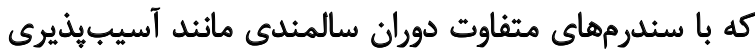

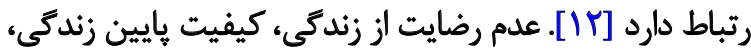

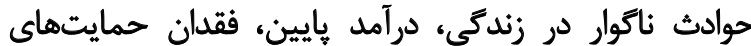

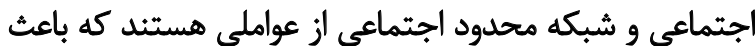

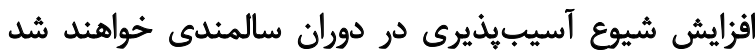

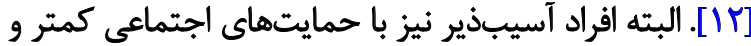

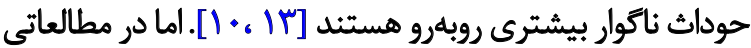

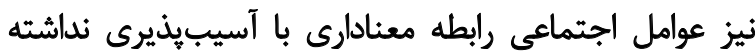

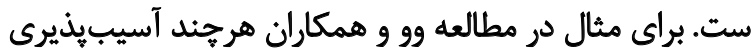

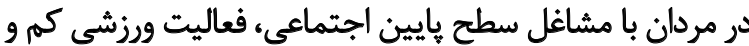

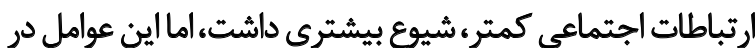

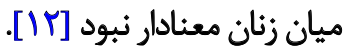

همجنين سندرم آسيبذئيرى در افراد داراى اختيلالات

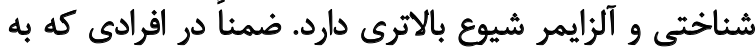

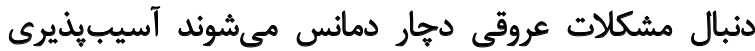




$$
\text { جريان بازدمى "19 است. }
$$

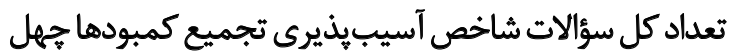

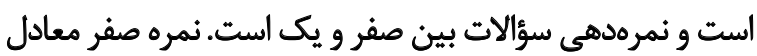

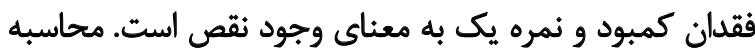

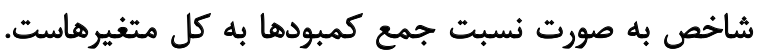

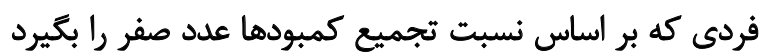

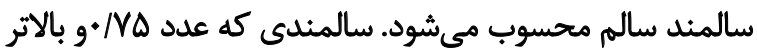

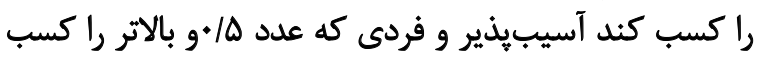

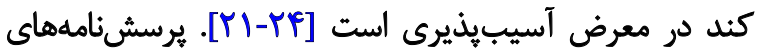

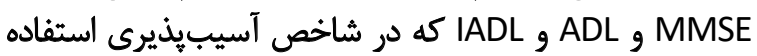

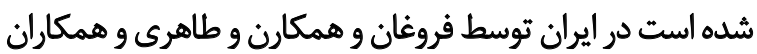

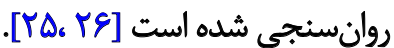

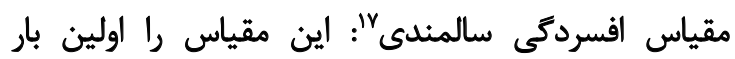

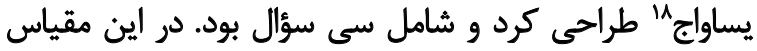

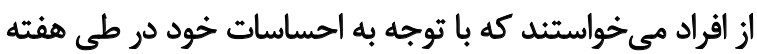

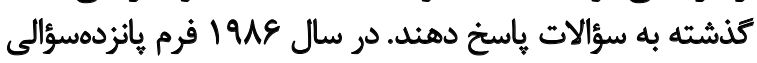

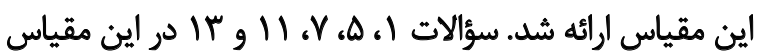

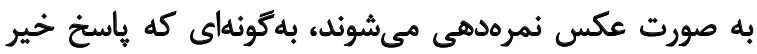

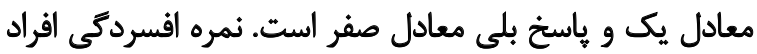

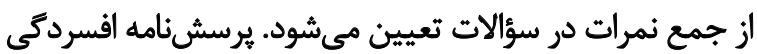

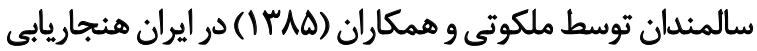

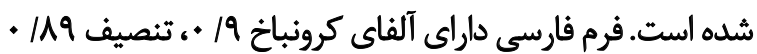

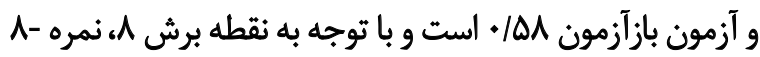
Q بيانكر افسردگى خفيف، نمره

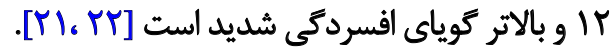

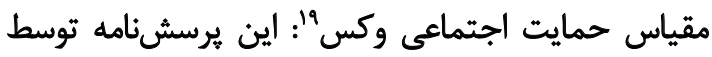

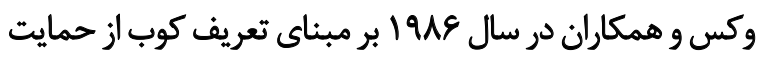

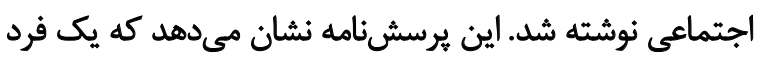

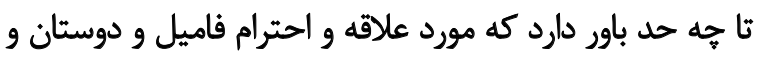

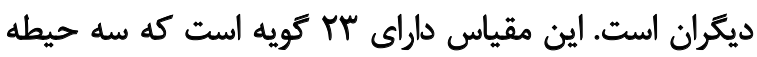

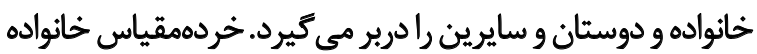

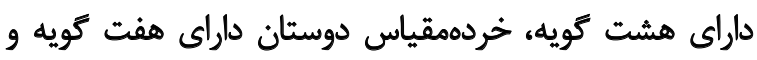

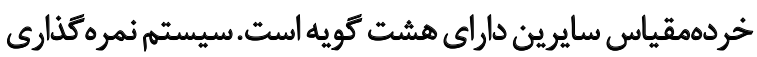

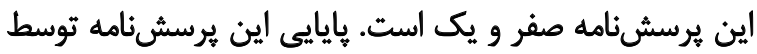

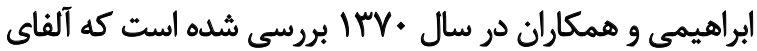

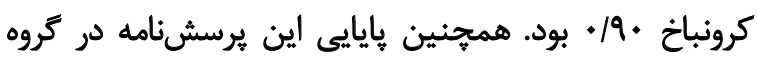

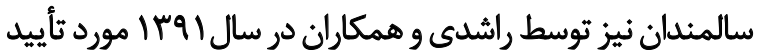

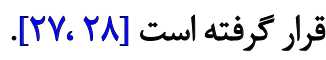

16. Peak flow

17. Geriatric Depression scale

18. Yesavage

19. Wax Social Support scale
انجام طرح يا تكميل ناقص سؤالات يرسشنامه بود. ابزارهاى جمع آورى دادها، جكىليست اطلاعات جمعيتشناختى

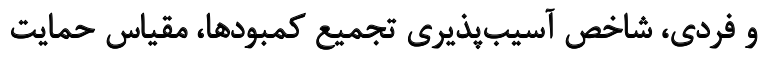

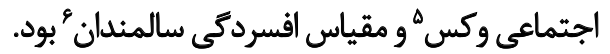

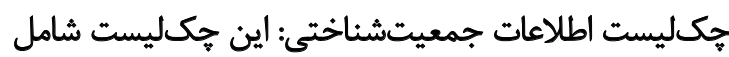

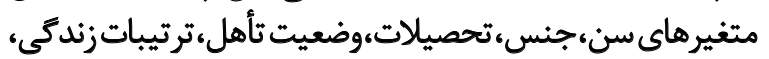

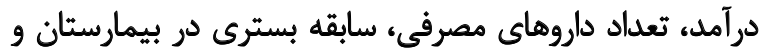
مصرف سيكاراست.

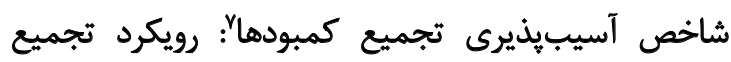

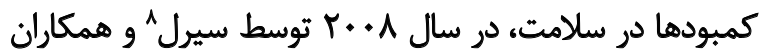

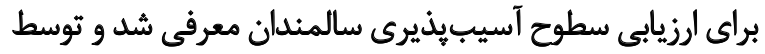

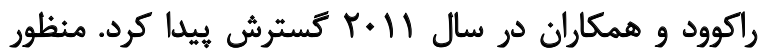

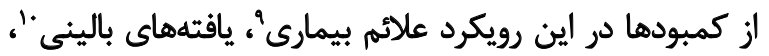

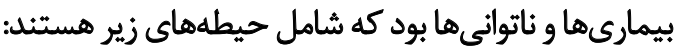

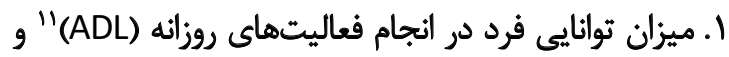

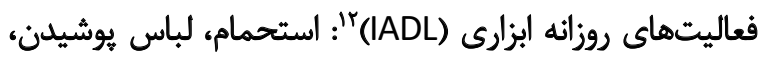

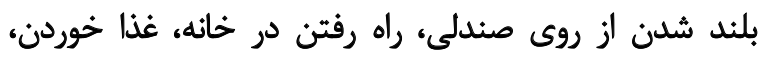

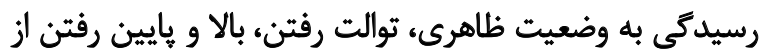

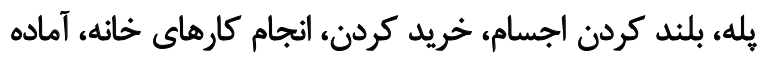
كردن غذا، مصرف دارو و انجام مسائل مالى.

ك.اختلال شناختى: نمره بهدستآمده از آزمون كوثاه وضعيت ذهنى

ب. ب. ارزيابى وضعيت سلامتى: سلامت خودارزياب، شاخص توده

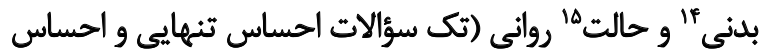

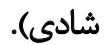

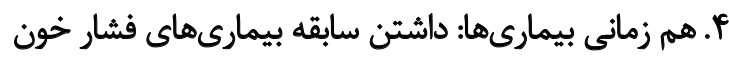

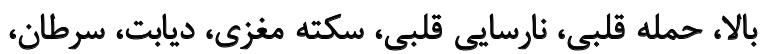
بيمارى مزمن ريوى و آرتريت.

ه. اختلال در عملكرد جسمانى: شامل راه رفتن سريع، راه رفتن معمولى، قدرت ميج دست، قدرت عضلات شانه و حداكثر رفئر

5. Wax Social Support scale

6. Geriatric Depression scale

7. Frailty Index of Accumulated Deficits

8. Searle

9. Symptoms

10. Signs

11. Activities of daily living

12. Instrumental activities of daily living

13. Mini-Mental State Examination (MMSE)

14. Body mass index

15. Mood 
از حمايتهاى اجتماعى ضعيف و فقط ألائ 19/4 درصد از حمايت اجتماعى بالايى برخوردار بودئد.

نتايج بررسى ارتباط عوامل جمعيتشناختى، مقياس حمايت

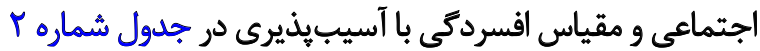

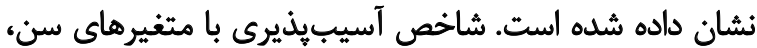

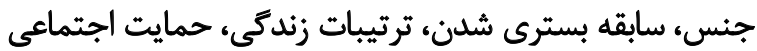

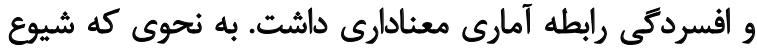

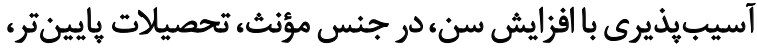

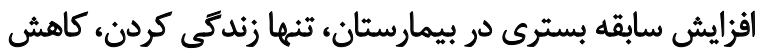

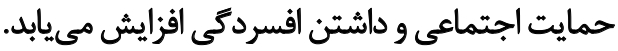

بين تعداد داروهاى مصرفى و شاخص آسيبيذيرى نيز

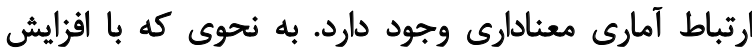

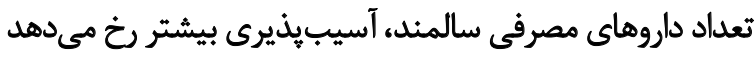

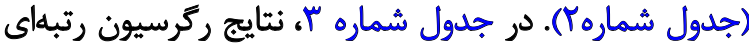

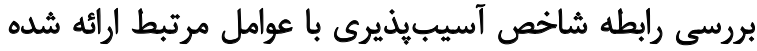

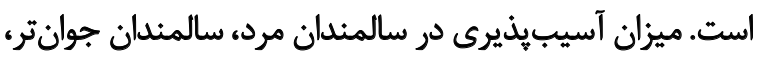

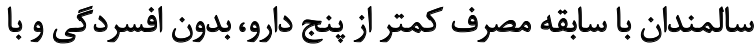

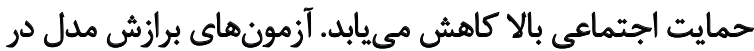

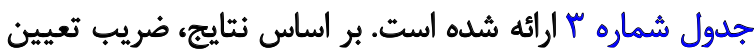

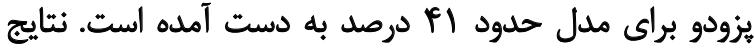

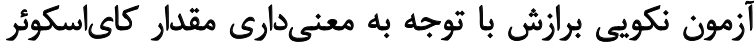

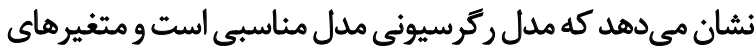

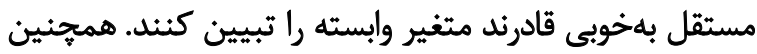

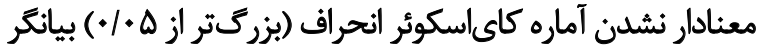

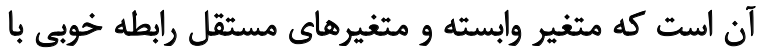

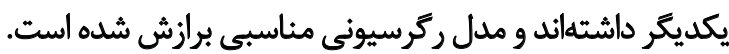

بحث

مطالعه حاضر با هدف تعيين عوامل مرتبط با آسيبذيذيرى

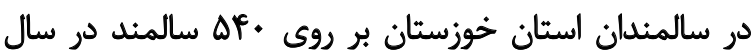

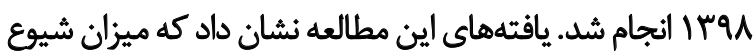

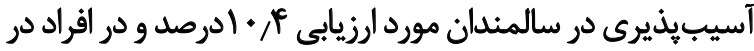

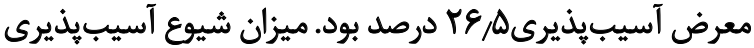

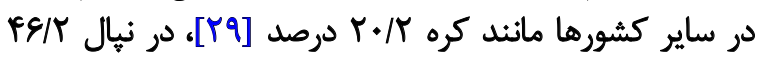

در اين تحقيق براي تجزيه و تحليل دادههاي جمع آورى نشاره

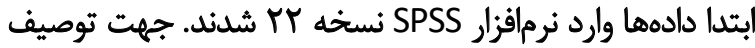

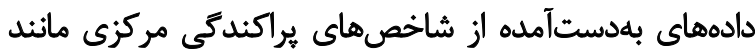

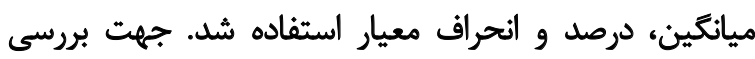

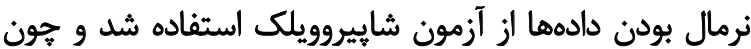

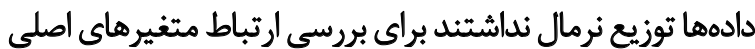

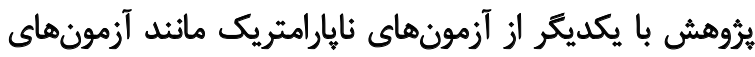

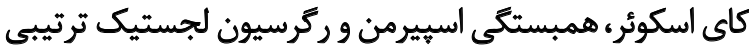

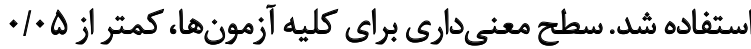
در نظر كرفته شد.

ياقتهها

در اين ثئوهش • هf سالمند مورد ارزيابى قرار كرفتند.

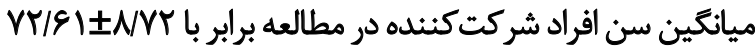

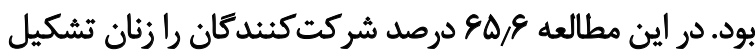

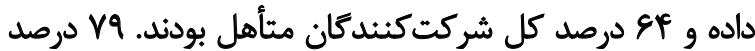

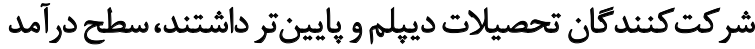

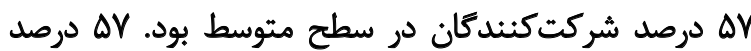

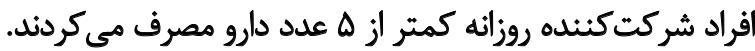

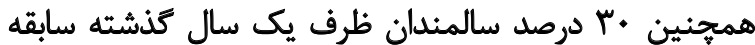

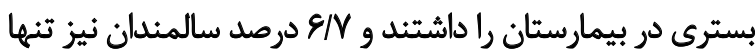

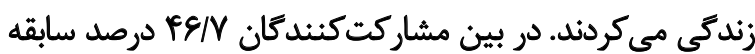

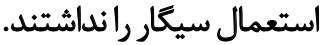

از كل سالمندان شركت كنينده در اين مطالعه، عر. •ا درصد

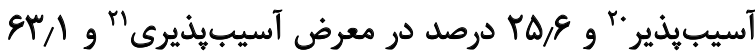

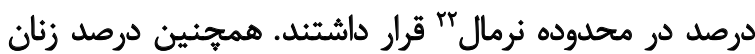

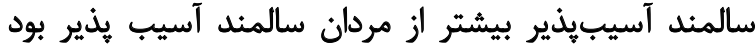

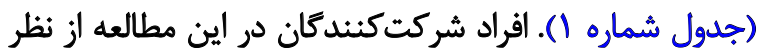

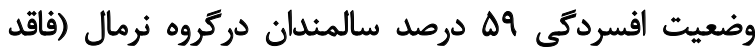

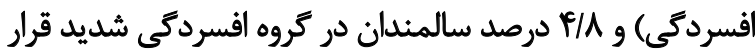

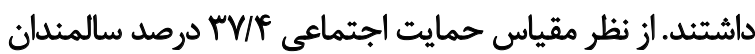

\section{Frail}

21. Pre-frail

22. Not frail

جدول ا. فراوانى و درصد سطوح شاخص آسيبيذيرى راكوود به تفكيك جنسيت

\begin{tabular}{|c|c|c|c|c|}
\hline \multicolumn{4}{|c|}{ تعداد (درصد)، سطح آسيبذيذيرى } & \multirow{2}{*}{ جنسيت } \\
\hline جمع كل & آسيبيذير & در معرض & 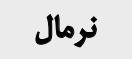 & \\
\hline$W E(r \varphi / \varphi)$ & $\operatorname{lf}(T / 9)$ & $\Delta \Delta(1+/ \pi)$ & $\| V(Y V / \Delta)$ & 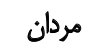 \\
\hline $\operatorname{rr\Delta }(\xi \Delta / \Omega)$ & $\operatorname{Rr}(\mathrm{V} / \Delta)$ & $M(1 S / T)$ & $\operatorname{Mr}(q) / \Delta)$ & زنان \\
\hline$\Delta f \cdot(1 \cdot)$. & $\Delta \&\left(1 \cdot / N^{\circ}\right)$ & $\ln (Y / \Delta / \Delta)$ & $\operatorname{re}(8 \pi / 1)$ & جمع كل \\
\hline
\end{tabular}

年 
جدول r. بررسى ارتباط بين عوامل مرتبط و سطوح آسيبيذيرى در ميان سالمندان استان خوزستان

\begin{tabular}{|c|c|c|c|c|c|c|}
\hline \multirow{2}{*}{$\mathbf{P}$} & \multirow{2}{*}{ آماره آزمون } & \multicolumn{3}{|c|}{ تعداد (درصد) } & \multirow{2}{*}{ مشخصه جمعيتى } & \multirow{2}{*}{ متغير } \\
\hline & & 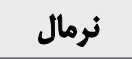 & در معرض & آسيبيذير & & \\
\hline \multirow{2}{*}{$.1 . .1$} & \multirow{2}{*}{$\chi^{r}=N / / r$} & $\operatorname{rre}(\theta) / \Delta)$ & $M(1 \& / M)$ & $\operatorname{Pr}(Y / D)$ & نن & \multirow{2}{*}{ جنس } \\
\hline & & $\| \gamma(\mid \Delta / \Psi)$ & $\Delta \Delta(1 \cdot / \pi)$ & $\operatorname{lf}(T / q)$ & مرد & \\
\hline \multirow{3}{*}{$<\cdot 1 . \cdot 1$} & \multirow{3}{*}{$\chi^{r}=r s / 1 r}$. & $\operatorname{Mr}(\mathrm{V \Delta} / \mathrm{T})$ & $g(T \cdot / \Lambda)$ & $M(r / q)$ & $\left(M^{2}-8 \cdot\right)$ & \multirow{3}{*}{ سن سن } \\
\hline & & $V Q(P V / A)$ & $\Delta P(M \varphi / \varphi)$ & YA(IVIA) & $(\lambda f-V \Delta)$ & \\
\hline & & $I V(T / M)$ & $M(r N \cdot)$ & $r V(F q / T)$ & $<A \Delta$ & \\
\hline \multirow{6}{*}{$.1 . \vee$} & \multirow{6}{*}{$\chi^{r}=r \mid r / I V$} & $r(\Delta / /)$ & $\operatorname{Ir}(Y \& M)$ & $1 .(T / T)$ & بيسواد & \multirow{6}{*}{ سطح تحصيلات } \\
\hline & & $F \cdot(\Delta Q / V)$ & $I V\left(T \Delta / T^{\prime}\right)$ & $1+(1) / 9)$ & ابتلايع & \\
\hline & & $\Delta r(\Delta r / q)$ & $r \Delta(r \Delta / A)$ & $r \cdot(r \cdot 19)$ & راهثمايق & \\
\hline & & $\Delta F(\Delta r / A)$ & $r(r V /)$ & $\mid Y(\mid \& / \cdot)$ & متوسطه & \\
\hline & & $r q(s q / 4)$ & $r \mu(r \cdot T)$ & $I r(1+/ \Delta)$ & دييلم & \\
\hline & & $v^{\varphi}(\varepsilon g M)$ & Tq(TE/) & $A(N / T)$ & انشكاهى & \\
\hline \multirow{3}{*}{$.1 .+1$} & \multirow{3}{*}{$\chi^{r}=\mu F / M A$} & $1 \cdot \Delta(\Delta Y / \cdot)$ & $\Delta Y(Y \Delta / N)$ & $P A(Y Y / Y)$ & ضعيف & \multirow{3}{*}{ حمايت اجتماعى } \\
\hline & & $10 \cdot(9 \times / 9)$ & $\Delta Q(T \Delta / \%)$ & $r \varphi(1 . / r)$ & متوسط & \\
\hline & & $s q(9 \Delta M)$ & rM(YI/V) & $A(N / 9)$ & خوب & \\
\hline \multirow{2}{*}{$<.1 . .1$} & \multirow{2}{*}{$X^{r}=r q q / r q$} & $r Y g(M / 1)$ & $\Delta q(1 N 9)$ & $\pi(1 \cdot / r)$ & ن نلارد & \multirow{2}{*}{ افسردگى } \\
\hline & & $q(\varphi+/))$ & $\Lambda \cdot(\Psi \varepsilon / \cdot)$ & $\mu(19 / 1)$ & دارد & \\
\hline \multirow{4}{*}{$<\cdot 1 .+1$} & \multirow{4}{*}{$\chi^{r}=-r \mid \Delta /}$. & $\mid q(\varphi F / F)$ & $q(T \Delta)$ & $M(r+)$ & تنها & \multirow{4}{*}{ ترتيبات زندكى } \\
\hline & & $\Delta E(f \Delta / q)$ & $r \Delta(T N Y)$ & $r(r \Delta / f)$ & فقط با همسر & \\
\hline & & $1 .+(E \& M)$ & $M e(T r / T)$ & $\mid V(\mid Y / 1)$ & با همسر و فرزئدان & \\
\hline & & $1 \Delta+(9 \Delta / \Delta)$ & SI(rg/G) & IAN/q) & فقط با فرزندان & \\
\hline \multirow{2}{*}{$<.1 . .1$} & \multirow{2}{*}{$\chi^{r}=99 / r^{\prime}$} & $1 \cdot N(\Delta T / \Delta)$ & $P q(T F / T)$ & $P \Delta(T T / M)$ & بلدون همسر & \multirow{2}{*}{ وضعيت تأهل } \\
\hline & & $119(9 / / 9)$ & $9 \cdot(Y \& / 8)$ & $\pi(q / \Delta)$ & داراى همسر & \\
\hline \multirow{6}{*}{ ( } & & $r(\Delta \mu r / \Delta)$ & $r+(T N T)$ & $\mathbb{I r}(\mathbb{N} \Psi)$ & كمتر از ا ميليون & \\
\hline & & $r q(\Delta T / Y)$ & $r V(r \cdot M)$ & $I Q(I V)$ & ا اتا ه/1 ميليون & \\
\hline & $\gamma^{\gamma}=r / M$ & $\operatorname{sr}(\xi \cdot / 1)$ & $r \cdot(r M)$ & $r \Delta(\mid N)$ & ها تا Y ميليون & درأمد سريرست خانوار \\
\hline & $\alpha \quad$ on & $\mathscr{S H}(S \& / M)$ & $r(r T / M)$ & $11(11 / 8)$ & r تا ه/T ميليون & (تومان) \\
\hline & & PN(PE) & $M(Y A)$ & $g(A)$ & ه/ه تا T ميليون & \\
\hline & & $F(q \pi)$ & $r \cdot(r V / F)$ & $V(q / q)$ & بيشتر از ك ميليون & \\
\hline & & $r(\varepsilon / /)$ & $19(r \Delta / \Gamma)$ & $r(w / Y)$ & ندارد & \\
\hline$<.1 . .1$ & $\chi^{r}=1 \otimes N$. & $\mid F N(F D / T)$ & $S N(T / T)$ & $M(N E)$ & $\Delta-1$ & تعداد مصرف دارو \\
\hline & & $\operatorname{Irr}(\Delta r / q)$ & $\Delta r(M T)$ & $\Delta r(T M)$ & ييشتر از ه دارو & \\
\hline$<\cdot 1 . \cdot 1$ & $x^{r}=1 f / M$ & $\Lambda \cdot(\Re N A)$ & $r \cdot(I N T)$ & $\Delta \Delta(T / / T)$ & 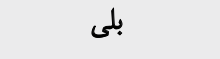 & ساقيه ستى ع \\
\hline & & $\operatorname{MrY}\left(g_{D} / 1\right)$ & $1.9(r 9 / 1)$ & $r r(\Delta / q)$ & خير & لسبعه بسرىى \\
\hline & $\chi^{\kappa}=. \%$ & $94(8 \cdot)$ & $P V(r \cdot)$ & $10(9 / M)$ & دارد & سابقه استعمال سيخار \\
\hline & & (E) & $9 T(T r / 9)$ & $\operatorname{Er}(1 \notin /)$ & ندارد & \\
\hline
\end{tabular}


جدول r. ركرسيون رتبهاى بين شدت آسيبدئيرى و عوامل مرثبط با آن در ميان سالمندان استان خوزستان

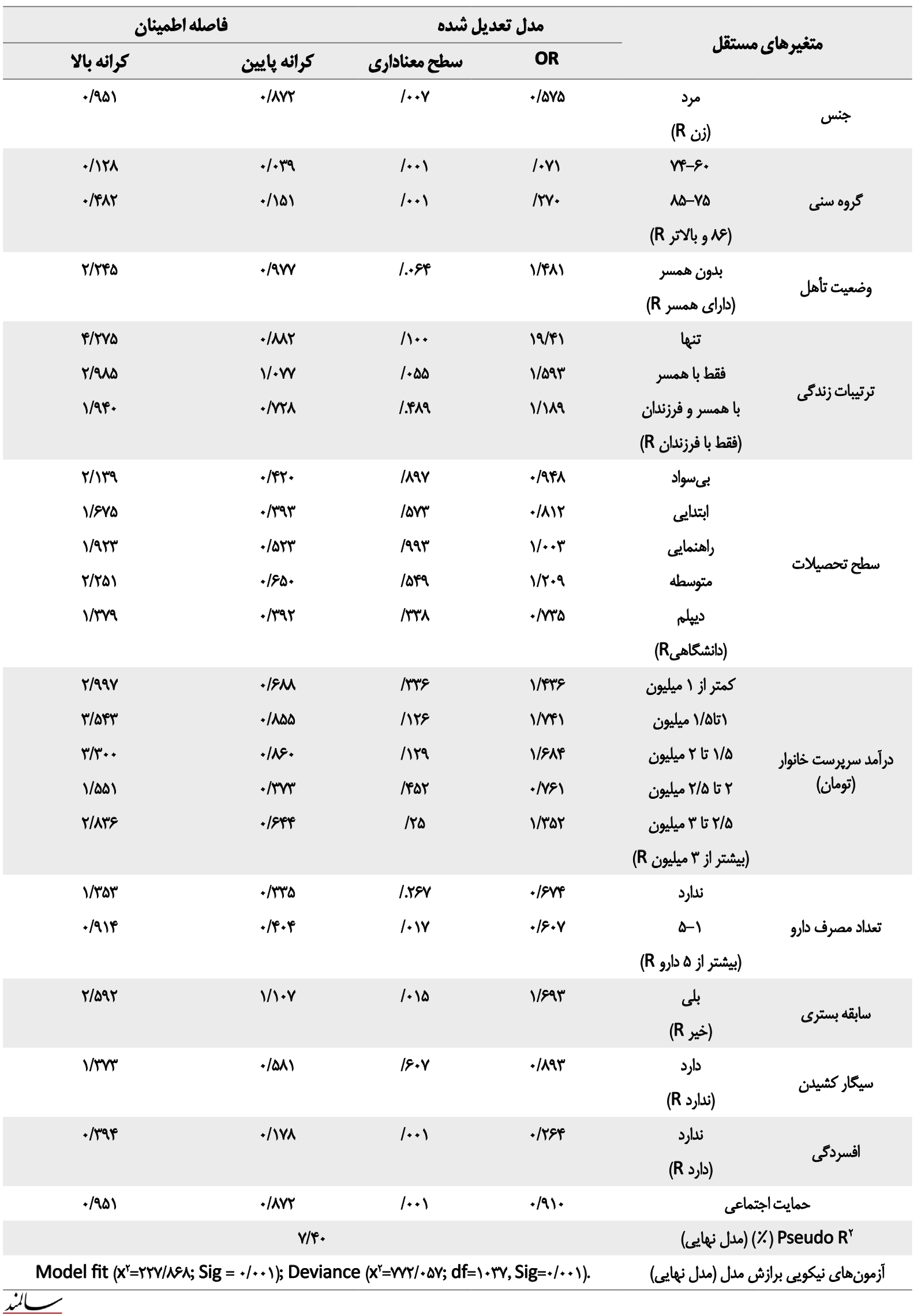


آسيبذيذيرى سالمندان در اين مطالعه بود. در ساير مطالعات نيز

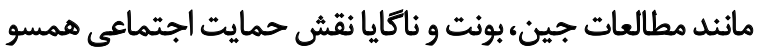

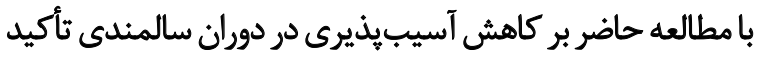

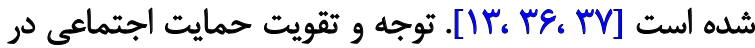
دوران سالمندى مىتواند در كاهش آسيب تيذيرى مؤثر باشئ. يكى ديكر از متغيرهاي مهم دركاهش آسيبيذيرى وضعيت

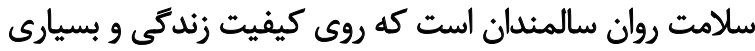

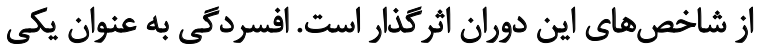

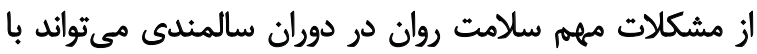

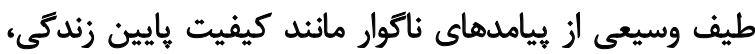

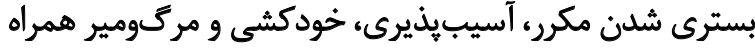

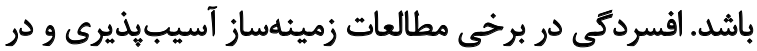

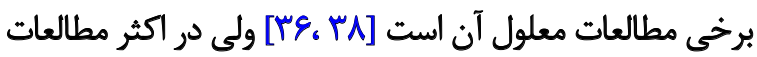

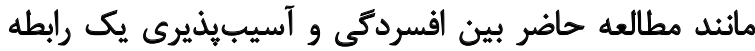

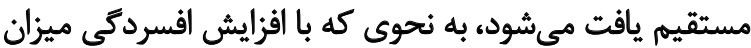

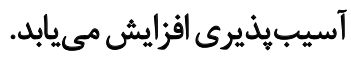

درنهايت اين نكته قابل توجه است كه آسيبيذيرى و عوامل

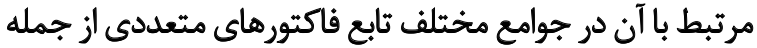

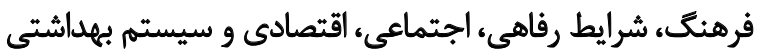

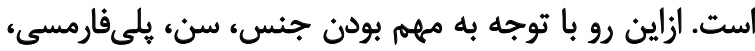

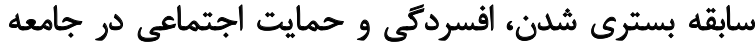

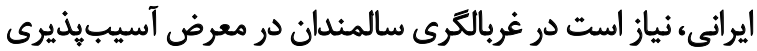

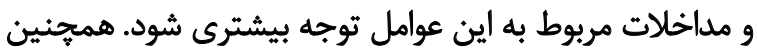

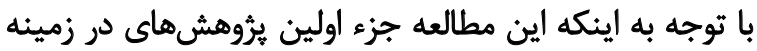

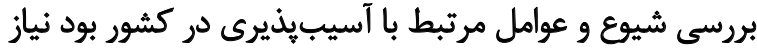

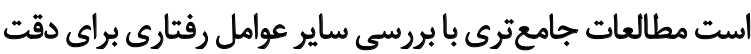
در ارائه خدمات به سالمندان انجام شود.

\section{تتيجلمَيرى نهايى}

با توجه به اينكه سندرم آسيبذيذيرى در دوران سالمندى

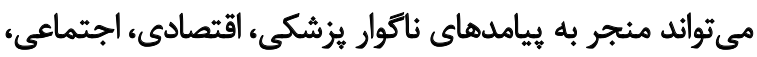

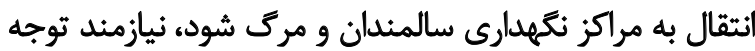

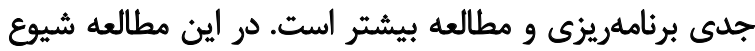

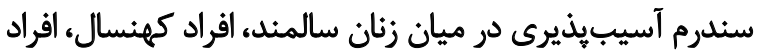

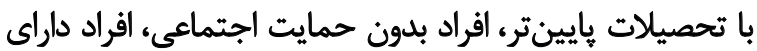

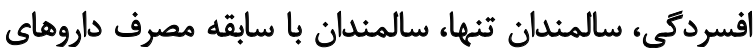

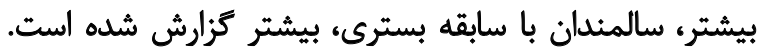

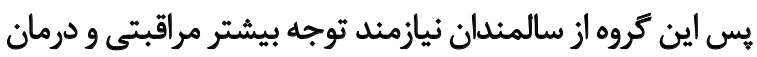

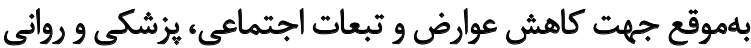

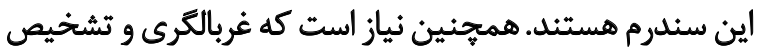

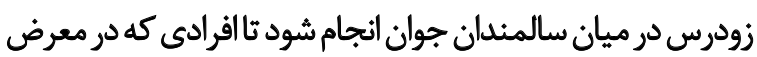

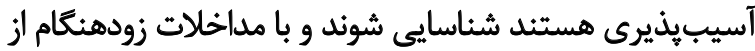

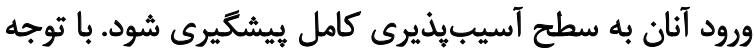

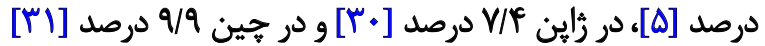

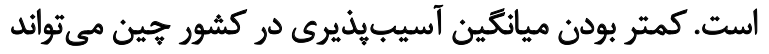

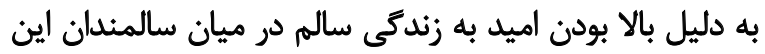

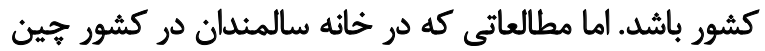

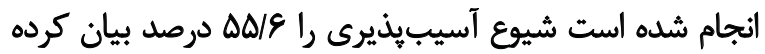

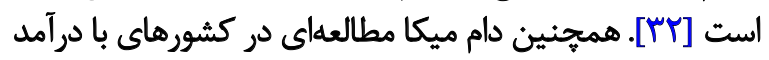

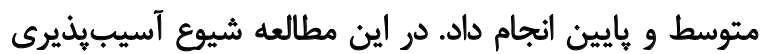

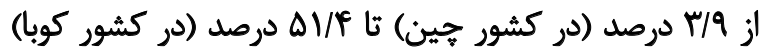

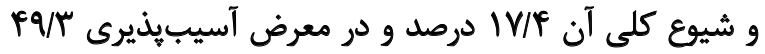

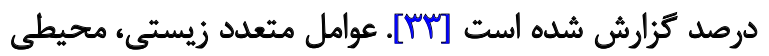

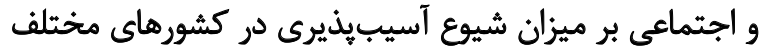

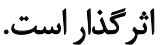

در مطالعات كشورها و شهرهاى مختلف ميزان ارتباط

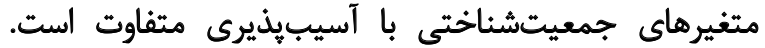

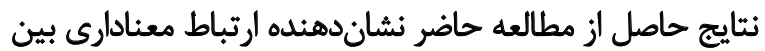

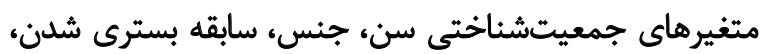

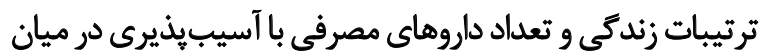

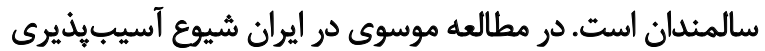

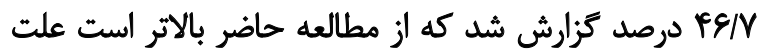

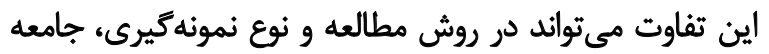

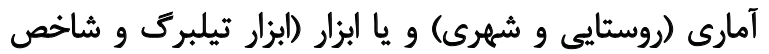

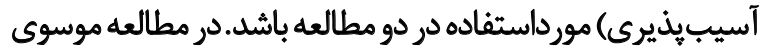

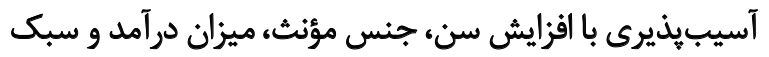

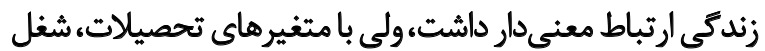

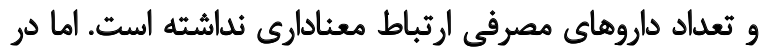

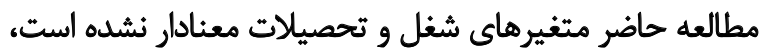

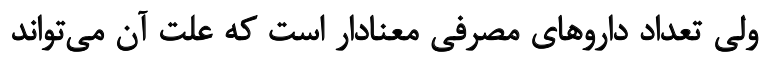

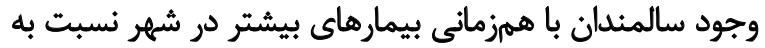

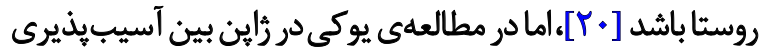

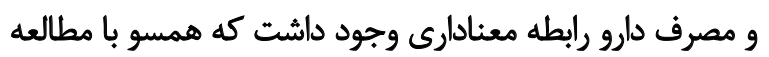

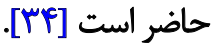

ساير مطالعات مانند مطالعه ليو در كشور جين و مطالعه راشما

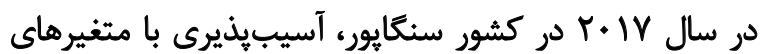

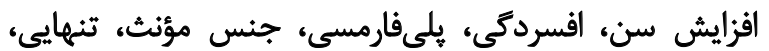

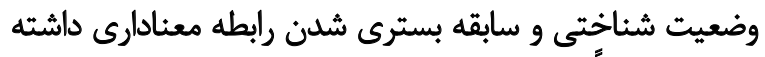

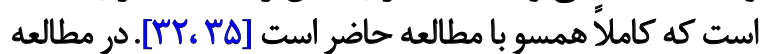

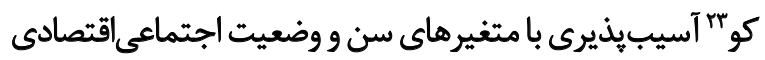

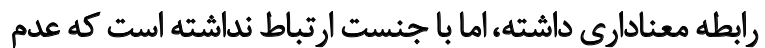

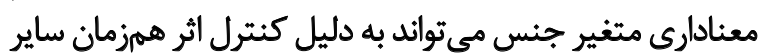

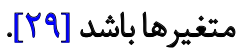
حمايت اجتماعى يكى از مههمرين متغيرهاي تأثيركذار بر 
به اينكه مطالعه حاضر يكى از اولين مطالعاتى است كه در ايران

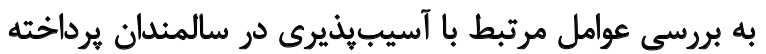

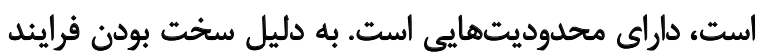

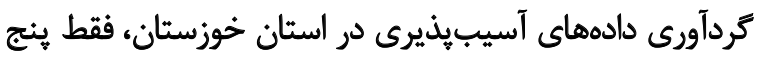

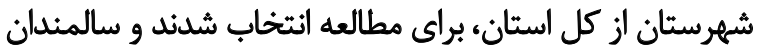

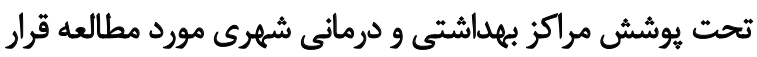

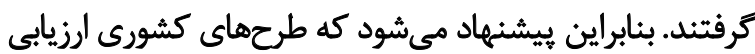

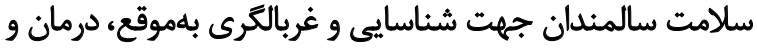

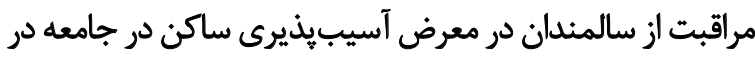

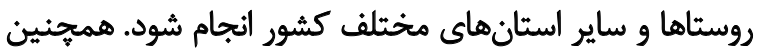

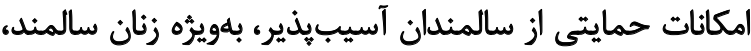

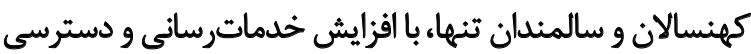

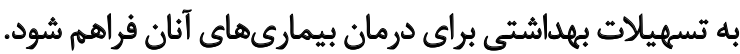

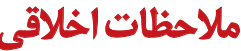

\section{ييروى از اصول اخلاق بئوهش}

اين مطالعه در كميته اخلاق دانشكاه علوم توانبخشى و سلامت

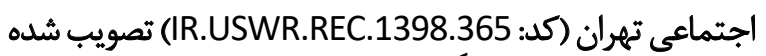

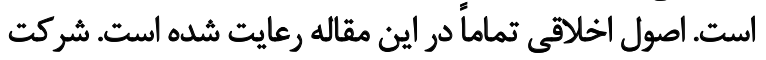

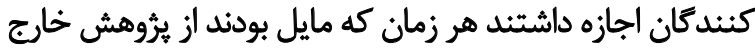

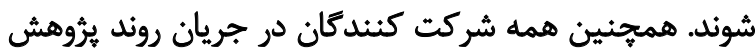

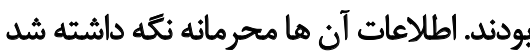

$$
\text { حامي مالي }
$$

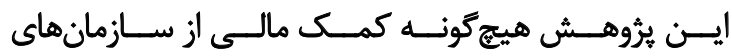

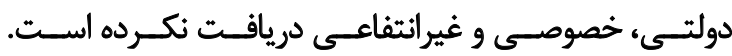

$$
\text { مشاركت نويسند مكان }
$$

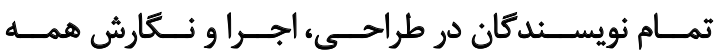

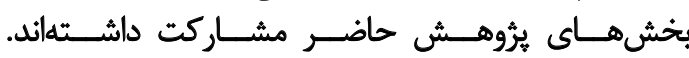

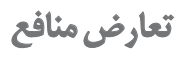

بنابر اظهار نويسندكان اين مقاله تعارض منافع ندارد.

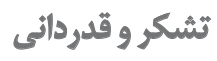

از تمامى استادان محترم گروه سالمندشناسى دانشكاه علوم

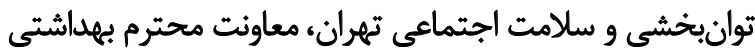

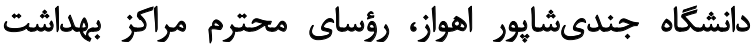

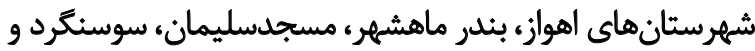

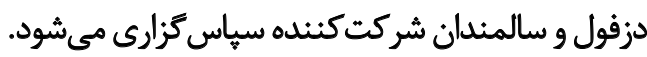




\section{References}

[1] Fillit H, Rockwood K, Young JB. Brocklehurst's textbook of geriatric medicine and gerontology. Amsterdam: Elsevier; 2016. https://www.elsevier.com/books/brocklehursts-textbook-ofgeriatric-medicine-and-gerontology/fillit/978-0-7020-6185-1

[2] Halter JB, Ouslander JG, Tinetti M, Studenski S, High KP, Asthana S. Hazzard's geriatric medicine and gerontology: New York City: McGraw Hill Professional; 2009. https://books.google. com/books?id=nFOHHXz9MI8C\&dqhttps:/ / accessmedicine. mhmedical.com/book.aspx?bookid=371

[3] Mitnitski AB, Rutenberg AD, Farrell S, Rockwood K. Aging, frailty and complex networks. Biogerontology. 2017; 18(4):433-46. [DOI:10.1007/s10522-017-9684-x]

[4] Rockwood K. What would make a definition of frailty successful? Age and Ageing. 2005; 34(5):432-4. [DOI:10.1093/ageing/ afi146]

[5] Devkota S, Anderson B, Soiza RL, Myint PK. Prevalence and determinants of frailty and associated comorbidities among older Gurkha welfare pensioners in Nepal. Geriatrics \& Gerontology International. 2017; 17(12):2493-9. [DOI:10.1111/ggi.13113]

[6] Vu HTT, Nguyen TX, Nguyen TN, Nguyen AT, Cumming R, Hilmer S, et al. Prevalence of frailty and its associated factors in older hospitalised patients in Vietnam. BMC Geriatrics. 2017; 17:216. [DOI:10.1186/s12877-017-0609-y]

[7] Grden CRB, Lenardt MH, de Sousa JAV, Kusomota L, Dellaroza MSG, Betiolli SE. [Associations between frailty syndrome and sociodemographic characteristics in long-lived individuals of a community (English-Spanish-Portuguese)]. Revista LatinoAmericana de Enfermagem. 2017; 25:e2886. [DOI:10.1590/15188345.1770.2886]

[8] de Carvalho Mello A, Engstrom EM, Alves LC. Health-related and socio-demographic factors associated with frailty in the elderly: A systematic literature review. Cadernos de Saúde Pública. 2014; 30(6):1143-68. [DOI:10.1590/0102-311X00148213]

[9] Gobbens RJJ, van Assen MALM, Luijkx KG, Wijnen-Sponselee MT, Schols JMGA. Determinants of frailty. Journal of the American Medical Directors Association. 2010; 11(5):356-64. [DOI:10.1016/j.jamda.2009.11.008]

[10] Ocampo-Chaparro JM, Reyes-Ortiz CA, Castro-Flórez X, Gómez F. [Frailty in older adults and their association with social determinants of health. The SABE Colombia study (EnglishSpanish)]. Colombia Médica. 2019; 50(2):89-101. [DOI:10.25100/ cm.v50i2.4121]

[11] Weiss CO. Frailty and chronic diseases in older adults. Clinics in Geriatric Medicine. 2011; 27(1):39-52. [DOI:10.1016/j. cger.2010.08.003]

[12] Woo J, Goggins W, Sham A, Ho SC. Social determinants of frailty. Gerontology. 2005; 51(6):402-8. [DOI:10.1159/000088705]

[13] Bunt S, Steverink N, Olthof J, van der Schans CP, Hobbelen JSM. Social frailty in older adults: A scoping review. European Journal of Ageing. 2017; 14(3):323-34. [DOI:10.1007/s10433-0170414-7]

[14] de Morais Fabrício D, Chagas MHN, Diniz BS. Frailty and cognitive decline. Translational Research. 2020; 221:58-64. [DOI:10.1016/j.trsl.2020.01.002]
[15] Petridou E. Cognitive frailty: A brief review. Journal of Research and Practice on the Musculoskeletal System. 2020; 4(4):113 24. http://www.jrpms.eu/articles/jrpms_v04i04_113.pdf

[16] Soysal P, Veronese N, Thompson T, Kahl KG, Fernandes BS, Prina AM, et al. Relationship between depression and frailty in older adults: A systematic review and meta-analysis. Ageing Research Reviews. 2017; 36:78-87. [DOI:10.1016/j.arr.2017.03.005]

[17] Ribeiro O, Duarte N, Teixeira L, Paúl C. Frailty and depression in centenarians. International Psychogeriatrics. 2018; 30(1):115-24. [DOI:10.1017/S1041610217001910]

[18] Alencar MA, Dias JMD, Figueiredo LC, Dias RC. Frailty and cognitive impairment among community-dwelling elderly. Arquivos de Neuro-Psiquiatria. 2013; 71(6):362-7. [DOI:10.1590/0004-282X20130039]

[19] Santos-Eggimann B, Cuénoud P, Spagnoli J, Junod J. Prevalence of frailty in middle-aged and older community-dwelling Europeans living in 10 countries. The Journals of Gerontology: Series A. 2009; 64A(6):675-81. [DOI:10.1093/gerona/glp012]

[20] Mousavisisi M, Shamshirgaran SM, Rezaeipandari H, Matlabi H. Multidimensional approach to frailty among rural older people: Applying the tilburg frailty indicator. Elderly Health Journal. 2019; 5(2):92-101. [DOI:10.18502/ehj.v5i2.2155]

[21] Rockwood K, Mitnitski A. Frailty defined by deficit accumulation and geriatric medicine defined by frailty. Clinics in Geriatric Medicine. 2011; 27(1):17-26. [DOI:10.1016/j.cger.2010.08.008]

[22] Rockwood K, Song X, MacKnight Ch, Bergman H, Hogan DB, McDowell I, et al. A global clinical measure of fitness and frailty in elderly people. CMAJ. 2005; 173(5):489-95. [DOI:10.1503/ cmaj.050051]

[23] Searle SD, Mitnitski A, Gahbauer EA, Gill TM, Rockwood K. A standard procedure for creating a frailty index. BMC Geriatrics. 2008; 8:24. [DOI:10.1186/1471-2318-8-24]

[24] Mitnitski AB, Mogilner AJ, Rockwood K. Accumulation of deficits as a proxy measure of aging. The Scientific World Journal. 2001; 1:321027. [DOI:10.1100/tsw.2001.58]

[25] Foroughan M, Jafari Z, Shirin Bayan P, Ghaem Magham Farahani Z, Rahgozar M. [Validation of Mini-Mental State Examination (MMSE) in the elderly population of Tehran (Persian)]. Advances in Cognitive Sciences. 2008; 10(2):29-37. http://icssjournal. ir/article-1-422-fa.html

[26] Taheri Tanjani P, Azadbakht M. [Psychometric properties of the Persian version of the activities of daily living scale and instrumental activities of daily living scale in elderly (Persian)]. Journal of Mazandaran University of Medical Sciences. 2016; 25(132):10312. http://jmums.mazums.ac.ir/article-1-6766-en.html

[27] Ebrahimi B, Hosseini M, Rashedi V. The relationship between social support and death anxiety among the elderly. Elderly Health Journal. 2018; 4(2):37-42. [DOI:10.18502/ehj.v4i2.261]

[28] Rashedi V, Rezaei M, Gharib M, Nabavi SH. [Social support for the elderly: Comparison between home and nursing home (Persian)]. Journal of North Khorasan University of Medical Sciences. 2013; 5(2):351-6. [DOI:10.29252/jnkums.5.2.351]

[29] Ko Y, Choi K. Prevalence of frailty and associated factors in Korean older women: The KLoSA study. Journal of Women \& Aging. 2017; 29(1):15-25. [DOI:10.1080/08952841.2015.1018069] 
[30] Kojima G, Iliffe S, Taniguchi Y, Shimada H, Rakugi H, Walters K. Prevalence of frailty in Japan: A systematic review and meta-analysis. Journal of Epidemiology. 2017; 27(8):347-53. [DOI:10.1016/j.je.2016.09.008]

[31] Ma L, Tang Zh, Zhang L, Sun F, Li Y, Chan P. Prevalence of frailty and associated factors in the community-dwelling population of China. Journal of the American Geriatrics Society. 2018; 66(3):559-64. [DOI:10.1111/jgs.15214]

[32] Liu W, Puts M, Jiang F, Zhou Ch, Tang S, Chen S. Physical frailty and its associated factors among elderly nursing home residents in China. BMC Geriatrics. 2020; 20:294. [DOI:10.1186/ s12877-020-01695-5]

[33] Siriwardhana DD, Hardoon S, Rait G, Weerasinghe MC, Walters KR. Prevalence of frailty and prefrailty among communitydwelling older adults in low-income and middle-income countries: A systematic review and meta-analysis. BMJ Open. 2018; 8(3):e018195. [DOI:10.1136/bmjopen-2017-018195]

[34] Yuki A, Otsuka R, Tange Ch, Nishita Y, Tomida M, Ando F, et al. Polypharmacy is associated with frailty in Japanese community-dwelling older adults. Geriatrics \& Gerontology International. 2018; 18(10):1497-500. [DOI:10.1111/ggi.13507]

[35] Merchant RA, Chen MZ, Tan LWL, Lim MYD, Ho HK, van Dam RM. Singapore Healthy Older People Everyday (HOPE) study: Prevalence of frailty and associated factors in older adults. Journal of the American Medical Directors Association. 2017; 18(8):734.E9-14. [DOI:10.1016/j.jamda.2017.04.020]

[36] Jin Y, Si H, Qiao X, Tian X, Liu X, Xue QL, et al. Relationship between frailty and depression among community-dwelling older adults: The mediating and moderating role of social support. The Gerontologist. 2020; 60(8):1466-75. [DOI:10.1093/geront/ gnaa072]

[37] Nagai K, Tamaki K, Kusunoki H, Wada Y, Tsuji Sh, Itoh M, et al. Physical frailty predicts the development of social frailty: A prospective cohort study. BMC Geriatrics. 2020; 20:403. [DOI:10.1186/s12877-020-01814-2]

[38] Vieira RA, Guerra RO, Giacomin KC, de Souza Vasconcelos KS, de Souza Andrade AC, Pereira LSM, et al. [Prevalence of frailty and associated factors in community-dwelling elderly in Belo Horizonte, Minas Gerais State, Brazil: Data from the FIBRA study (Portuguese)]. Cadernos de Saúde Pública. 2013; 29(8):1631-43. [DOI:10.1590/S0102-311X2013001200015] 
This Page Intentionally Left Blank 